\title{
Croissance et caractérisation de graphène au Pôle CNFM de Lille
}

\author{
S. Ben Salk, E. Pallecchi, V. Hoel, H. Happy
}

Pôle CNFM de Lille - Université de Lille, Villeneuve d'Ascq, France

Contacts email : henri.happy@univ-lille.fr

Le graphène en particulier, et les matériaux 2D en général, sont une nouvelle filière de matériaux aux propriétés physiques très intéressantes. Leurs propriétés électroniques, thermiques et mécaniques en font des matériaux de choix pour de nombreuses applications, dans les domaines aussi que les technologies de l'information et de la communication, la santé, l'énergie, le transport. Compte tenu des potentialités de ces matériaux, de nombreux grands programmes de recherche se sont développés au niveau international, pour étudier ces matériaux, et bénéficier des innovations qui en découleraient pour le développement de nouveaux emplois et de nouvelles richesses. Sur le plan de la formation, avec le soutien du GIP CNFM et de l'IDEFI FINMINA obtenu par le réseau du CNFM, le pôle CNFM de Lille s'est doté de nouveaux équipements qui permettent d'explorer, d'analyser, et de fabriquer des composants et systèmes avec ces matériaux 2D. Il s'agit notamment d'un four de croissance de graphène, d'un spectromètre Raman permettant de caractériser les matériaux 2D, du développement d'un système de transfert du graphène sur un matériau hôte. Cette communication va présenter la méthodologies mise en place, pour sensibiliser les étudiants du master 2 sur la manipulation de ces nouveaux matériaux.

\section{Introduction}

Isolé pour la première fois en 2004 à partir du graphite par André Geim et Constantin Novoselov (1), le graphène se présente sous la forme d'un matériau bi-dimensionnel constitué d'une monocouche de carbone ( $c f$. Fig 1). Un matériau bidimensionnel (2D) se caractérise par le fait que toutes les liaisons de covalences sont orientées dans le même plan, et ne présente pas de liaison de covalence dans un plan perpendiculaire. Ces matériaux $2 \mathrm{D}$ ont des propriétés physiques particulières, et c'est la démonstration de ces propriétés qui a permis à A. Geim et K. Novoselov d'obtenir le prix Nobel de physique en 2010.

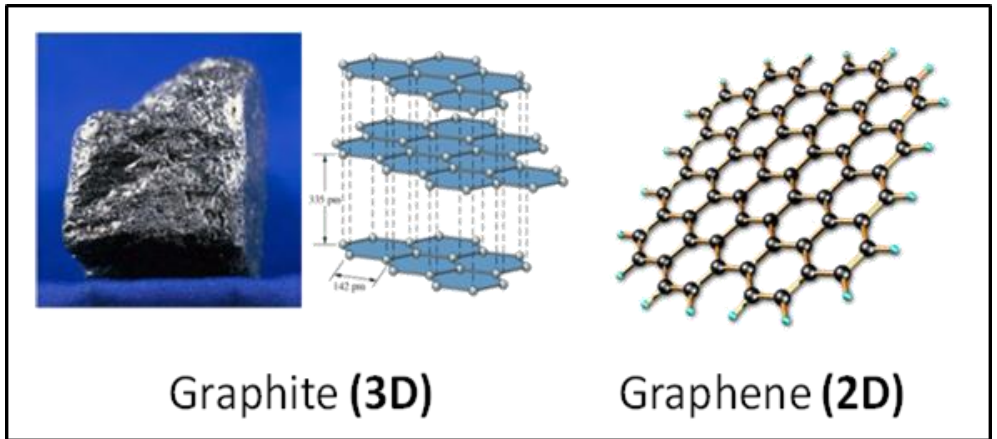

Fig.1. Illustration d'une monocouche de graphène 2D isolée à partir du graphite (matériau 3D) constitué de plans de graphène, sans liaison de covalence entre eux. 
Depuis sa découverte en 2004, le graphène a suscité un engouement auprès des chercheurs, car il s'est avéré être un matériau de choix pour mener de nombreuses études. En effet le graphène, du point de vue théorique, est un système électronique bidimensionnel parfait. Il n'est composé que d'une seule couche d'atomes de carbone disposés dans un réseau en nid d'abeille, la dynamique des porteurs de charge est donc nécessairement confinée dans un système extrêmement fin. Les études menées sur ce matériau ont permis de mettre en évidence ses propriétés particulières en termes de transport électronique, de propriétés optiques, de propriétés mécaniques, thermiques, chimiques.

A titre d'illustration, la mobilité intrinsèque du graphène peut atteindre une valeur de l'ordre de $2.10^{5} \mathrm{~cm}^{2} / \mathrm{Vs}$ à température ambiante (2-3) ; Ce qui en fait un matériau de choix pour l'électronique rapide. Le graphène est un matériau léger, pesant seulement environ 0,77 milligrammes par mètre carré. De par son épaisseur, il est flexible et assez transparent. La transparence d'un film de graphène de $2 \mathrm{~nm}$ d'épaisseur est de $95 \%$, et reste supérieure à $70 \%$ pour un film de $10 \mathrm{~nm}$ d'épaisseur (4). Le graphène peut être utilisé par exemple pour la fabrication d'électrodes transparentes, pour des applications dans le domaine de la récupération d'énergie. Le graphène est un matériau très résistant, qui présente un module de Young entre 0.5 et $1 \mathrm{TPa}$ (5). Il présente ainsi un intérêt pour le renforcement des matériaux composites (6) ou pour la réalisation de capteurs extrêmement sensibles.

En se basant sur les différentes propriétés du graphène, la figure 2 illustre les grands domaines d'applications du graphène, qui touchent de nombreux secteurs industriels.

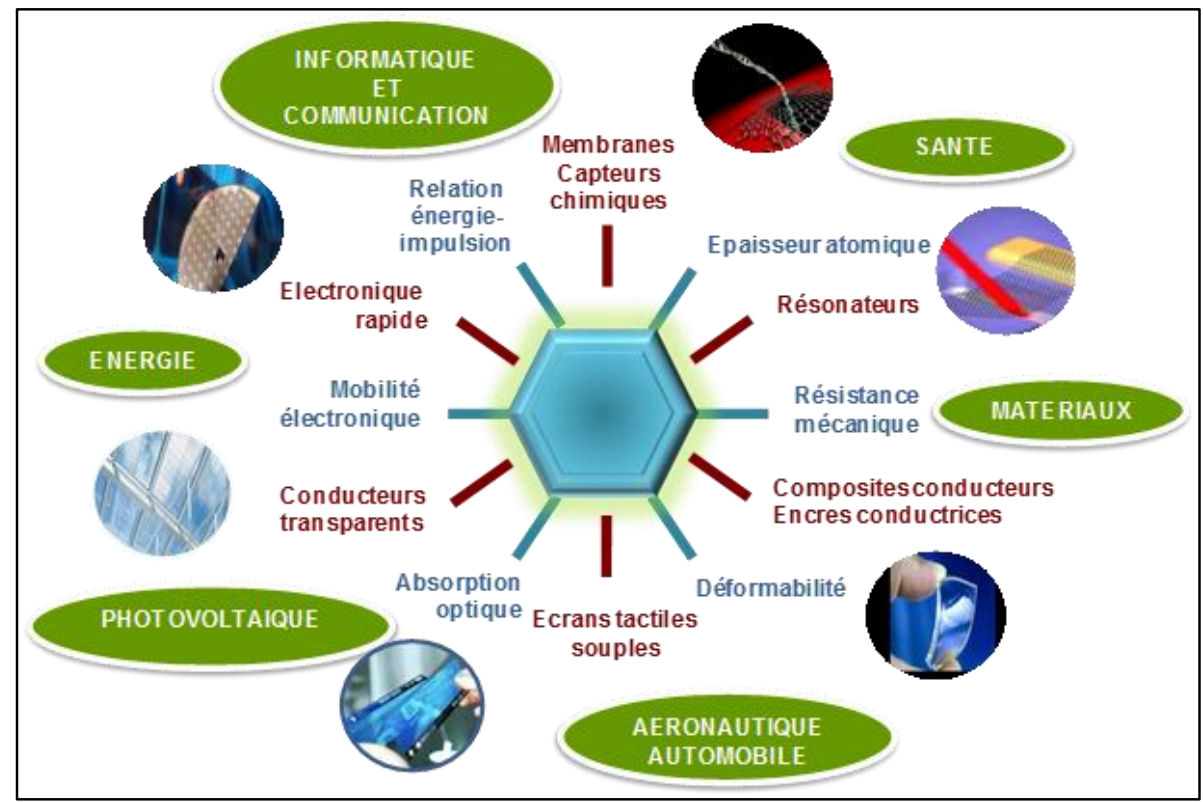

Fig.2. Illustration des propriétés du graphène, des applications qui en découlent, ainsi que les grands secteurs pouvant être impactés par ce nouveau matériau.

\section{Croissance du graphène}

Depuis la découverte du graphène par exfoliation mécanique, plusieurs techniques ont été mises au point pour synthétiser des matériaux de grande surface (2-3). Parmi ces différentes techniques, pour nos besoins, nous avons opté pour la croissance par CVD (Chemical Vapor Deposition) sur feuillet de cuivre. Le principe de base consiste à introduire dans un four adapté (illustration sur la figure 2-a) un précurseur de carbone sous forme gazeuse tel que le méthane $\left(\mathrm{CH}_{4}\right)$, un gaz neutre tel que l'argon (Ar) pour équilibrer 
la pression de croissance, et un gaz actif tel que l'hydrogène $\left(\mathrm{H}_{2}\right)$ pour réguler la vitesse de croissance. La réaction chimique mise en place sous l'effet de la température, à la surface du cuivre, est du type :

$$
\mathrm{CH}_{4} \leftrightarrow \mathrm{C}+2 \mathrm{H}_{2}
$$

L'optimisation des paramètres de croissance a été réalisée dans le cadre du stage de master de M. Daoudi AMINE (2017), sur le four de croissance dédié à la formation (Fig. 3-b). Il s'agit d'un four de type NanoCVD de faible volume, facile à manipuler, et pouvant accueillir des substrats de cuivre de dimension maximale $2 \mathrm{~cm} \times 3 \mathrm{~cm}$.

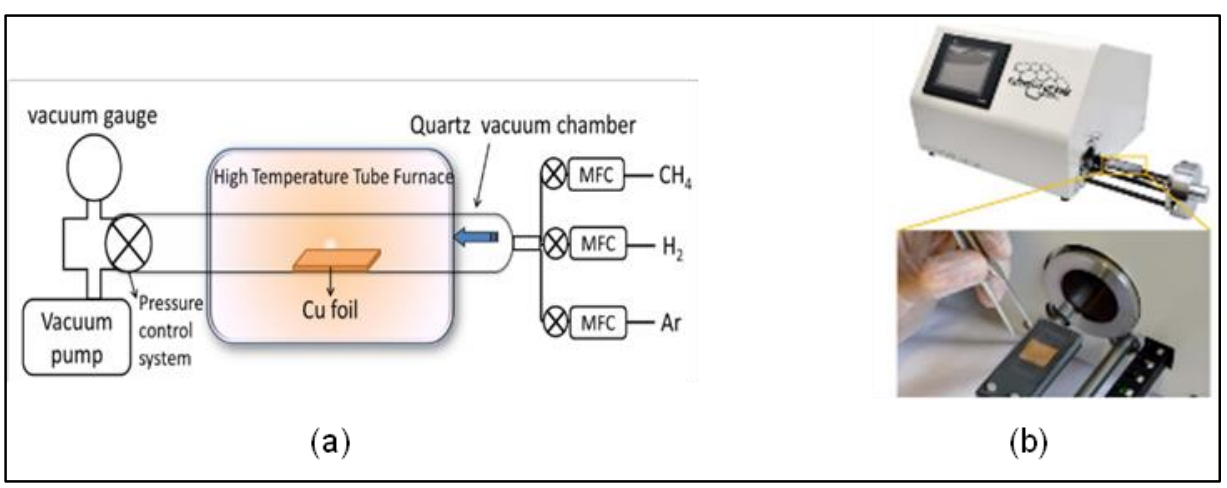

Fig.3. Four de croissance CVD du graphène : a) Principe de croissance du graphène par CVD ; b) Photo du four de croissance du graphène du pôle CNFM de Lille.

\section{III.Caractérisation du graphène après croissance}

L'une des méthodes de caractérisation du graphène la plus couramment utilisée est la spectroscopie Raman. C'est une méthode rapide et non destructive, très utilisée pour déterminer les propriétés structurales des matériaux à base de carbone (9-10). Les différents pics du graphène renseignent notamment sur la qualité du matériau, le nombre de couches de graphène, la nature des contraintes, etc. Dans le cadre de la mise en place de ce nouveau travail pratique (TP) innovant, nous avons acquis un spectromètre de marque Horiba Xplora équipé d'un kit laser $532 \mathrm{~nm}$. Cet équipement, illustré sur la figure 4-a, permet d'obtenir le spectre Raman du graphène synthétisé sur cuivre.

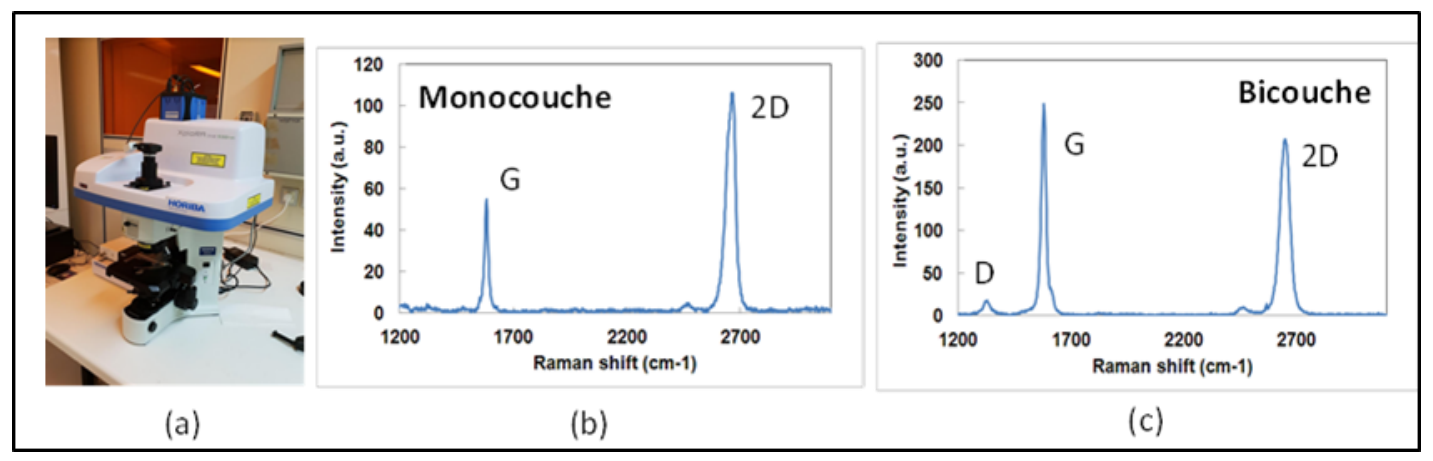

Fig.4. Spectromètre Raman de type Horiba Xplora disponible pour la caractérisation non destructive du graphène (a) ; Spectres Raman du graphène sur cuivre dans 2 zones différentes représentant une structure monocouche (b); ou une structure bicouche (a).

La Figure 4-b-c montre le spectre Raman mesuré sur la couche de graphène, sur 2 zones différentes. L'axe Y représente une unité arbitraire de l'intensité du signal Raman. On retrouve sur ces courbes les résonnances typiques du graphène, avec un pic $\mathrm{D}$ autour $1300 \mathrm{~cm}^{-1}$, qui caractérise le taux de défaut dans le matériau; un pic $\mathrm{G}$ autour de 
$1500 \mathrm{~cm}^{-1}$ et un pic 2D autour de $2700 \mathrm{~cm}^{-1}$ caractérisant la couche de graphène. Le ratio des intensités des pics $\mathrm{I}_{2 \mathrm{D}} / \mathrm{I}_{\mathrm{G}}$ donne une estimation du nombre de couches dans la zone d'étude. Ainsi pour $\mathrm{I}_{2 \mathrm{D}} / \mathrm{I}_{\mathrm{G}}>1$, on a une monocouche de graphène (Fig. 4-b), tandis que pour $\mathrm{I}_{2 \mathrm{D}} / \mathrm{I}_{\mathrm{G}}<1$, on s'oriente vers des structures multicouches (Fig. 4-c). Ici il s'agit d'une zone constituée d'une structure bicouche de graphène.

\section{IV.Transfert du graphène sur un substrat hôte}

La croissance du graphène est effectuée sur un substrat métallique. Il est donc nécessaire, si l'on souhaite utiliser la couche de graphène, de le transférer sur un substrat adapté à l'application visée. La méthode de transfert décrite ici a été mise au point par Mme Soukaina BEN SALK, dans le cadre de son stage de master en 2016, et qui poursuit sa thèse dans notre groupe de recherche. Pour effectuer un transfert rapide, dans la durée d'une séance de TP, nous avons choisi de mettre au point la technique de transfert par électrochimie, dont le principe est illustré sur la figure 5.

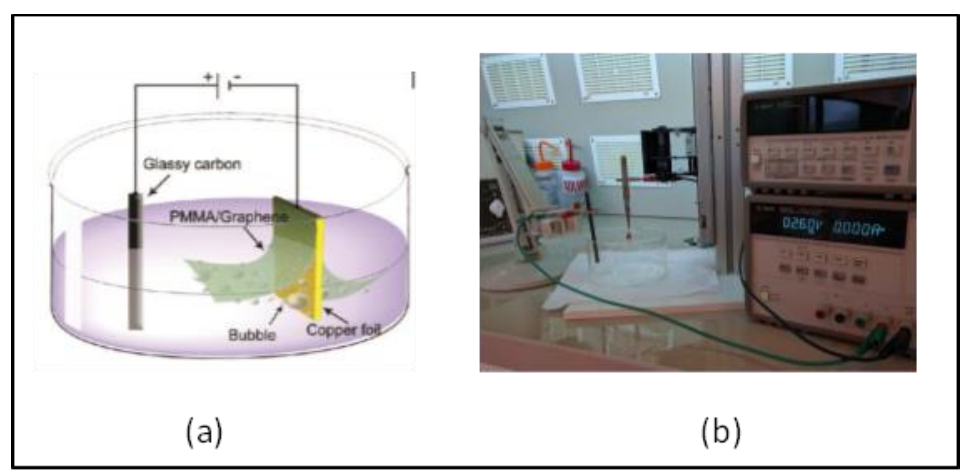

Fig.5. Principe du transfert par électrochimie (a), et dispositif expérimental développé (b).

Un courant continu (CC) est appliqué entre l'électrode «polymère/graphène/cuivre » et une autre électrode, réalisée en platine ou en carbone vitreux, dans une cellule électrolytique. Les solutions d'électrolyte comprennent les sels de sodium ou de potassium tels que $\mathrm{NaOH}, \mathrm{KOH}, \mathrm{NaCl}, \mathrm{KCl}$, etc. Une tension négative est alors appliquée sur la feuille de $\mathrm{Cu}$, ce qui provoque la génération de bulles d' $\mathrm{H}_{2}$ à l'interface cuivre-graphène en raison du procédé de décomposition de $\mathrm{H}_{2} \mathrm{O}$ :

$$
2 \mathrm{H}_{2} \mathrm{O}(l) \rightarrow \mathrm{H}_{2}(g)+2 \mathrm{OH}^{-}(a q)
$$

Les bulles de $\mathrm{H}_{2}$ aident à séparer le graphène de la surface $\mathrm{Cu}$, d'où la dénomination « transfert du graphène en utilisant les bulles». Pour une surface de $1 \mathrm{~cm} \times 1 \mathrm{~cm}$, la durée de décollement du graphène de la surface du cuivre est inférieure à $5 \mathrm{mn}$.

Toute la procédure de transfert est décrite sur la figure 6 . En effet, après la croissance du graphène sur cuivre, on recouvre ce dernier d'une couche de résine de type polyméthacrylate de méthyl (poly-methyl-methacrylate ou PMMA). Cette résine servira de support pour le transfert, et permettra aussi de localiser le graphène dans la solution de transfert. En effet une fois décollée, la monocouche de graphène est transparente et invisible à l'œil nu. L'ensemble PMMA/graphène est décollé du cuivre en utilisant la solution électrolytique décrite précédemment. Cette structure bicouche PMMA/graphène flotte à la surface de la solution et peut être récupérée sur un substrat hôte. Dans ce cas de figure, un substrat silicium est utilisé. Des traitements de surface permettent de réduire le 
piégeage de bulles d'eau entre le silicium et le graphène. Après une étape de séchage, on peut éliminer la résine à la surface du graphène.

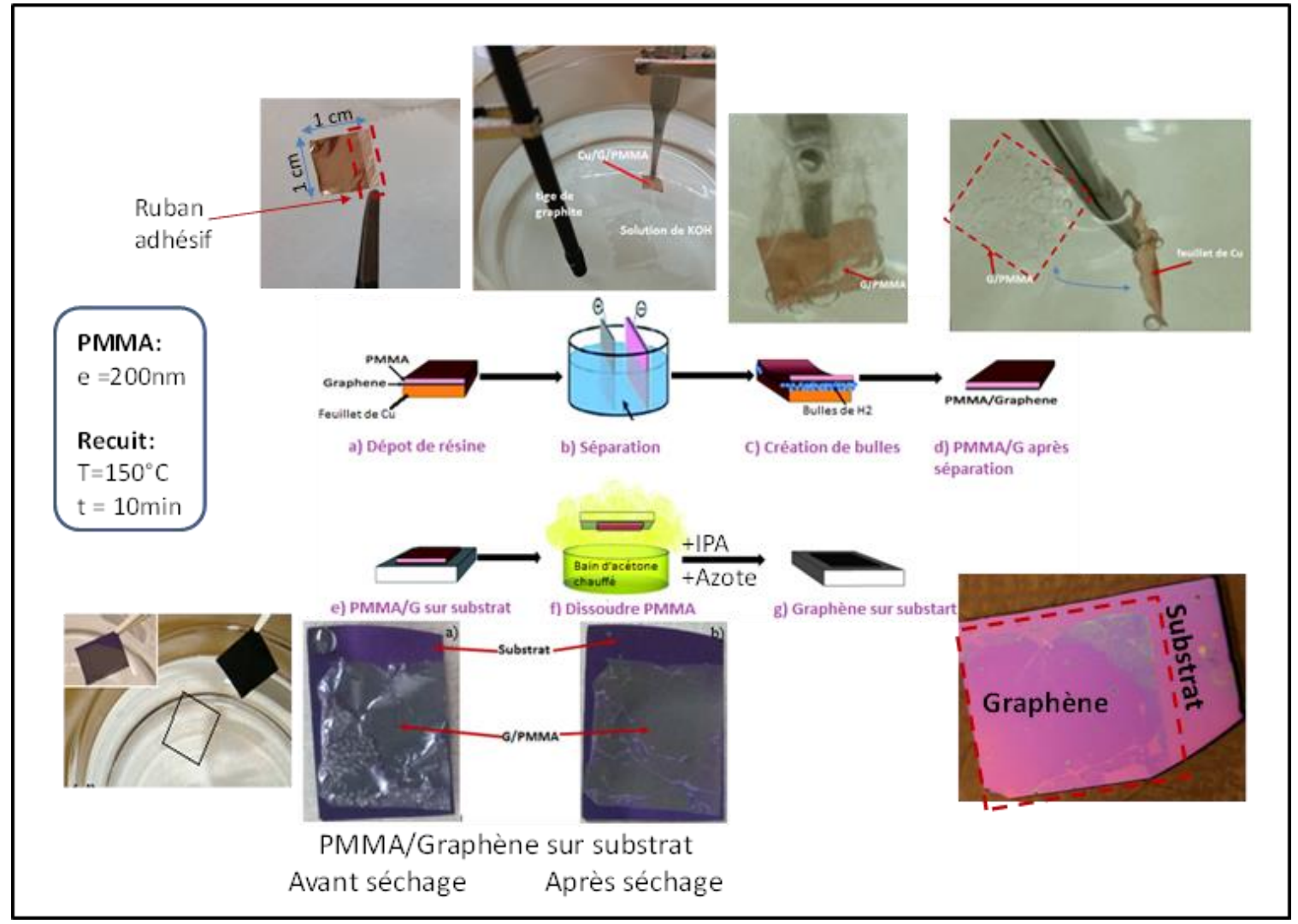

Fig.6. Procédure de transfert du graphène de la feuille de cuivre vers un substrat hôte (ici $\mathrm{SiO}_{2} / \mathrm{Si}$ ).

La caractérisation Raman de la couche transférée sur substrat silicium est illustrée sur la figure 7. Le spectre Raman montre, dans le cas d'une monocouche : un pic D à $1354 \mathrm{~cm}^{-1}$, un pic $\mathrm{G}$ à $1584 \mathrm{~cm}^{-1}$ et un pic $2 \mathrm{D}$ à $2703 \mathrm{~cm}^{-1}$. La légère augmentation du pic $\mathrm{D}$ traduit une légère augmentation du nombre de défauts (légère dégradation du matériau) après transfert du graphène, par rapport à l'état initial.

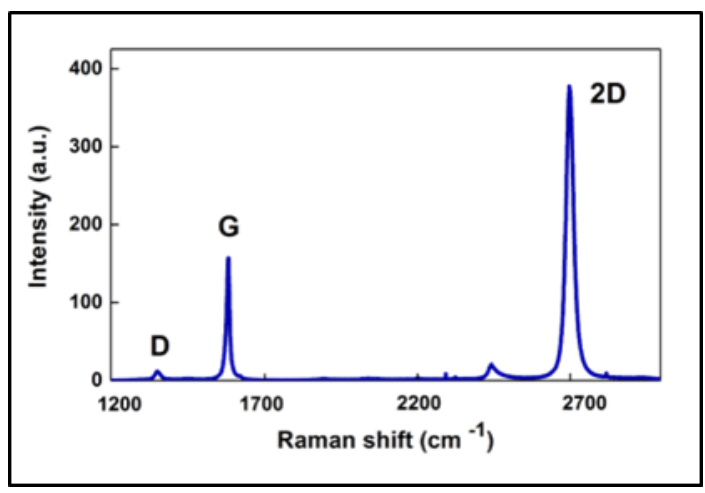

Fig.7. Spectre Raman du graphène obtenu par croissance CVD sur cuivre, après transfert sur substrat silicium.

\section{Conclusion}

Cette présentation explique la méthodologie mise en place pour sensibiliser les étudiants à la croissance, la manipulation et la caractérisation du graphène. La méthode développée 
ici s'applique non seulement au graphène, mais également à de nombreux autres matériaux 2D. En effet depuis la découverte du graphène, de nombreux matériaux lamellaires ont été découverts. C'est le cas notamment des matériaux de type dichalchogénures, qui présentent des nouvelles propriétés complémentaires du graphène (matériaux semi-conducteurs, isolants, magnétiques, etc.). De plus, la possibilité de fabriquer des hétérostructures de ces matériaux a augmenté les possibilités d'application des matériaux 2D.

\section{Remerciements}

Ce travail est soutenu grâce au financement du PIA FINMINA (ANR-11-IDFI-0017) (11), aux financements par projets du GIP CNFM (Groupement d'Intérêt Public pour la Coordination Nationale de la Formation en Microélectronique et en nanotechnologies) (12), et a également bénéficié du soutien de la fondation FACE (French American Cultural Exchange) par le biais du projet Partner University Fund (PUF - High frequency nanotube printed circuits).

\section{Références}

1. K. S. Novoselov, A. K. Geim, S. V. Morozov, D. Jiang, Y. Zhang, S. V. Dubonos, I. V. Grigorieva, and A. A. Firsov, "Electric field effect in atomically thin carbon films," Science, 306, 5696, pp. 666-669 (Oct. 2004).

2. S. V. Morozov, K. S. Novoselov, M. I. Katsnelson, F. Schedin, D. C. Elias, J. A. Jaszczak, and A. K. Geim, "Giant intrinsic carrier mobilities in graphene and its bilayer," Phys. Rev. Lett., 100(1), pp. 016 602-1-016 602-4 (2008).

3. K. Bolotin, K. Sikes, Z. Jiang, M. Klima, G. Fudenberg, J. Hone, P. Kim, and H. Stormer, "Ultrahigh electron mobility in suspended graphene," Solid State Commun., 146(9/10), pp. 351-355 (2008).

4. X. Wang, L. Zhi, K. Mullen, « Transparent, conductive graphene electrodes for dye-sensitized solar cells ». Nano Letters, 8(1):323-7 (2008).

5. C. Lee, X. Wei, JW. Kysar, J. Hone, « Measurement of the elastic properties and intrinsic strength of monolayer graphene ». Science, 321 (5887):385-8 (2008).

6. D. Cai, K. Yusoh, M. Song, «The mechanical properties and morphology of a graphite oxide nanoplatelet/polyurethane composite ». Nanotechnology, 20(8):085712 (2009).

7. C. Berger, Z. Song, T. Li, X. Li, A. Y. Ogbazghi, R. Feng, Z. Dai, A. N. Marchenkov, E. H. Conrad, P. N. First, and W. A. de Heer, "Ultrathin epitaxial graphite: 2D electron gas properties and a route toward graphene-based nanoelectronics," J. Phys. Chem. B, 108(52), pp. $19912-19916$ (2004).

8. C. Soldano, A. Mahmood, E. Dujardin, "Production, properties and potential of graphene"; CARBON, 48, pp. 2127-2150 (2010).

9. MS. Dresselhaus, G. Dresselhaus, A. Jorio, AG. Souza Filho, MA. Pimenta, R. Saito, « Single nanotube Raman spectroscopy ». Accounts Chem Res., 35(12):1070-8 (2002).

10. AC. Ferrari, JC. Meyer, V. Scardaci, C. Casiraghi, M. Lazzeri, F. Mauri, et al., « Raman Spectrum of Graphene and Graphene Layers ». Phys Rev Lett, 97(18):187401(2006).

11. IDEFI-FINMINA : Initiative d'Excellence - Formation Innovante en MIcroélectronique et Nanotechnologies, ANR-11-IDFI-0017. Website: http://www.cnfm.fr/VersionFrancaise/actualites/ FINMINA.htm

12. GIP-CNFM: Groupement d'Intérêt Public - Coordination Nationale pour la formation en Microélectronique et en nanotechnologies. Website: http://wwww.cnfm.fr 